\title{
Stage 0a Bladder Urothelial Carcinoma AJCC v6 and v7
}

National Cancer Institute

\section{Source}

National Cancer Institute. Stage Oa Bladder Urothelial Carcinoma A/CC v6 and v7. NCI Thesaurus. Code C9267.

Stage 0a includes: Ta, NO, MO. Ta: Papillary noninvasive carcinoma. NO: No regional lymph node metastasis. M0: No distant metastasis. (AJCC 6th and 7th eds.) 